\title{
Problem of Cauchy for linear singularly perturbed impulsive systems
}

Ljudmil Ivanov Karandjulov and Yana Petrova Stoyanova 


\title{
PROBLEM OF CAUCHY FOR LINEAR SINGULARLY PERTURBED IMPULSIVE SYSTEMS
}

\author{
Ljudmil Ivanov KARANDJULOV and Yana Petrova STOYANOVA \\ Department of Differential Equations, Technical University of Sofia \\ P.O.Box 384, Sofia 1000, Bulgaria \\ likar@vmei.acad.bg, yast@vmei.acad.bg
}

[Received: January 16, 2002]

\begin{abstract}
An initial value problem for singularly perturbed systems of ordinary differential equations is considered in a critical case. A unique asymptotic expansion of the solution is constructed by the method of boundary functions and generalized inverse matrices and projectors under some additional conditions.
\end{abstract}

Mathematical Subject Classification: 34B05, 34E15

Keywords: initial value problem, impulses, singularly perturbed systems, generalized inverse matrices and projectors

\section{Formulation of the problem}

A singularly perturbed system

$$
\begin{gathered}
\varepsilon \frac{d x}{d t}=A x+\varepsilon A_{1}(t) x+\varphi(t), t \in[a, b], t \neq \tau_{i}, i=\overline{1, p}, 0<\varepsilon<<1, \\
a \equiv \tau_{0}<\tau_{1}<\cdots<\tau_{p}<\tau_{p+1} \equiv b
\end{gathered}
$$

is considered. The coefficients of system (1.1) satisfy the following conditions

H1: A is $n \times n$ matrix with constant elements. It has an eigenvalue $\lambda=0$, whose multiplicity is $r$ and $r$ linear independent eigenvectors correspond to this eigenvalue. The remaining $(n-r)$ eigenvalues have negative real parts, i.e.,

$$
\lambda_{j} \in \sigma(A), \quad \operatorname{Re} \lambda_{j}<0, \quad j=\overline{1, n-r}, \quad \lambda_{j}=0, j=\overline{n-r+1, n} .
$$

Condition H1 shows that system (1.1) is considered in a critical case [9].

H2: $A_{1}(t)$ is $n \times n$ matrix. Its elements are continuously differentiable functions of class $C^{\infty}[a, b]$. 
H3: Vector-function $\varphi(t):[a, b] \rightarrow \mathbf{R}^{n}$ is partially continuous with break points of the first kind $\tau_{i}, i=\overline{1, p}$, i.e.,

$$
\begin{gathered}
\varphi(t)=\varphi_{i}(t), t \in\left(\tau_{i-1}, \tau_{i}\right], i=\overline{1, p+1}, \varphi(a)=\varphi_{1}\left(\tau_{0}\right), \\
\varphi(b)=\varphi_{p+1}\left(\tau_{p+1}\right), \quad \varphi_{i+1}\left(\tau_{i}\right)=\lim _{t \rightarrow \tau_{i}+0} \varphi(t), i=\overline{1, p} .
\end{gathered}
$$

An $n$-dimensional vector-function $x(t, \varepsilon)$ is sought for such that $x(\cdot, \varepsilon)$ is continuously differentiable in every subinterval $\left[a, \tau_{0}\right],\left(\tau_{i-1}, \tau_{i}\right], i=\overline{2, p+1}, x(t, \cdot) \in C\left(0, \varepsilon_{0}\right]$ and satisfying system (1.1), the generalized initial condition

$$
D x(a)=v
$$

and the generalized impulse conditions in fixed moments of time

$$
N_{i} x\left(\tau_{i}+0\right)+M_{i} x\left(\tau_{i}-0\right)=h_{i}, i=\overline{1, p} .
$$

The matrix $D$ is known $s \times n$ matrix with constant elements, $v$ is given column vector from $\mathbf{R}^{s}$ and $M_{i}, N_{i}, i=\overline{1, p}$, satisfy the following condition

H4: $M_{i}, N_{i}, i=\overline{1, p}$ are known $k_{i} \times n$ matrices with constant elements, $h_{i} \in \mathbf{R}^{k_{i}}$ are given column vectors.

If $\varepsilon=0$ in (1.1), then the degenerate system is obtained

$$
A x+\varphi(t)=0
$$

which has a solution

$$
x_{0}(t)=P_{A}^{r} \alpha_{0}(t)-A^{+} \varphi(t), t \in\left(\tau_{i-1}, \tau_{i}\right], i=\overline{1, p+1},
$$

if and only if

H5: $P_{A^{*}}^{r} \varphi(t)=0, t \in\left(\tau_{i-1}, \tau_{i}\right], i=\overline{1, p+1}$.

Here $\alpha_{0}(t)$ is a partially continuous arbitrary r-dimensional vector-function. $A^{+}$ denotes a unique Moore - Penrose inverse matrix of the matrix A. According to H1 $\operatorname{rank} A=n-r$, then $\operatorname{rank} P_{A}=\operatorname{rank} P_{A^{*}}=r$, where $P_{A}$ and $P_{A^{*}}$ are projectors

$$
P_{A}: \mathbf{R}^{n} \rightarrow \operatorname{ker} A, P_{A^{*}}: \mathbf{R}^{n} \rightarrow \operatorname{ker} A^{*}, A^{*}=A^{T} .
$$

In $n \times n$ matrix $P_{A}$ there exist $r$ linear independent columns and in $n \times n$ matrix $P_{A^{*}}$ there exist $r$ linear independent rows. $P_{A}^{r}$ denotes $n \times r$ matrix consisting of arbitrary $r$ linear independent columns of the matrix $P_{A}$ and $P_{A^{*}}^{r}$ denotes $r \times n$ matrix consisting of arbitrary $r$ linear independent rows of the matrix $P_{A^{*}}$.

The asymptotic expansion of the solution of the problem (1.1-1.3) is sought for so that under $\varepsilon \rightarrow 0$ it tends to solution (1.5) of the degenerate system (1.4) when $t \in\left(\tau_{i-1}, \tau_{i}\right] . i=\overline{1, p+1}$.

The essential methods for investigating linear and nonlinear impulsive systems are presented in [2], [7].

Initial and boundary-value problems for singularly perturbed systems of the kind

$$
\frac{d x}{d t}=f(t, x, y), \quad \varepsilon \frac{d y}{d t}=g(t, x, y)
$$


are considered in monography [8]. [9] considers a critical case for systems of the form $\varepsilon \frac{d x}{d t}=A(t) x+\varepsilon f(t, x, \varepsilon)$, with initial condition of the form $x(a)=v$. If impulse conditions of the form

$$
\left.\Delta x\right|_{t=\tau_{i}}=S_{i} x+a_{i}, \quad i=\overline{1, p}
$$

are added to (1.6), a system investigated in [1] is obtained. In the same monography initial problems for systems of the form

$$
\varepsilon \frac{d x}{d t}=f(t, x, \varepsilon)
$$

are considered in a critical case. In [7], [1] the fundamental matrix of solutions of the impulsive system

$$
\left.\frac{d x}{d t}=A(t) x, \quad t \neq \tau_{i},\left.\quad \Delta x\right|_{(} t=\tau_{i}\right)=S_{i} x, \quad i=\overline{1, p}
$$

is essential under condition $\operatorname{det}\left(S_{i}+E\right) \neq 0$. Making use of the fundamental matrix solutions of system (1.6), (1.7) and system (1.8), (1.7) are constructed in [1].

In the present work generalized impulse conditions are considered and additional requirements are not set for the matrices $M_{i}, N_{i}$. Therefore the fundamental matrix from [7], [1] can not be used in this case.

In this paper the method of boundary functions is used to construct an asymptotic expansion of the solution of the singular problem posed. The generalized inverse matrices and projectors are also used [4], [5], [6].

\section{Asymptotic expansion}

The asymptotic expansion of the solution of problem (1.1-1.3) is sought for in the form

$$
x(t, \varepsilon) \equiv x^{i}(t, \varepsilon)=\sum_{k=0}^{\infty} \varepsilon^{k}\left(x_{k}^{i}(t)+\Pi_{k}^{i}\left(\nu_{i}\right)\right), \quad \nu_{i}=\frac{t-\tau_{i-1}}{\varepsilon},
$$

where $t \in\left[\tau_{0}, \tau_{1}\right]$ and $t \in\left(\tau_{i-1}, \tau_{i}\right]$, under $i=\overline{2, p+1}$. The elements $x_{k}^{i}(t)$ of the expansion generate regular series and $\Pi_{k}^{i}\left(\nu_{i}\right)$ are boundary functions in the right neighborhood of the points $\tau_{i-1}, \quad i=\frac{1, p+1}{1, p}$ and generate singular series of the solution.

For the elements of the regular series the following systems are obtained

$$
\begin{aligned}
& A x_{0}^{i}(t)=-\varphi_{i}(t), \\
& A x_{k}^{i}(t)=\dot{x}_{k-1}^{i}(t)-A_{1}(t) x_{k-1}^{i}(t), \quad k=1,2,3, \cdots,
\end{aligned}
$$

and for the elements of the singular series - the systems

$$
\frac{d \Pi_{k}^{i}\left(\nu_{i}\right)}{d \nu_{i}}=A \Pi_{k}^{i}\left(\nu_{i}\right)+f_{k}^{i}\left(\nu_{i}\right), \quad k=0,1,2, \cdots, \quad i=\overline{1, p+1},
$$

where the functions $f_{k}^{i}\left(\nu_{i}\right), i=\overline{1, p+1}$ have the presentation

$$
f_{k}^{i}\left(\nu_{i}\right)=\left\{\begin{array}{cl}
0, & k=0 \\
\sum_{j=0}^{k-1} \frac{A_{1}^{(k-j-1)}\left(\tau_{i-1}\right)}{(k-j-1) !} \nu_{i}^{k-j-1} \Pi_{j}^{i}\left(\nu_{i}\right), & k=1,2, \cdots
\end{array}\right.
$$


Systems (2.2) have solutions of the form

$$
\begin{aligned}
& x_{0}^{i}(t)=P_{A}^{r} \alpha_{0}^{i}(t)-A^{+} \varphi_{i}(t), \\
& x_{k}^{i}(t)=P_{A}^{r} \alpha_{k}^{i}(t)+A^{+}\left(L x_{k-1}^{i}\right)(t), \quad k=1,2,3, \cdots,
\end{aligned}
$$

if and only if $\mathrm{H} 5$ and

(I): $P_{A^{*}}^{r}\left(L x_{k-1}^{i}\right)(t)=0, k=1,2,3, \cdots, i=\overline{1, p+1}$

are fulfilled. The condition of solvability (I) will be used for the determination of $r$ - dimensional vector - functions $\alpha_{k}^{i}(t), k=1,2,3, \cdots, i=\overline{1, p+1} .(L x)(t)=$ $\left(\frac{d}{d t} x-A_{1}(t) x\right)(t)$.

$X(t)$ denotes a normal fundamental matrix of solutions of the homogeneous system $\frac{d x}{d t}=A x$. According to condition H1 matrix $T$ exists such that

$$
\operatorname{det} T \neq 0 \quad \text { and } \quad A=T\left(\begin{array}{cc}
\bar{A} & 0 \\
0 & 0
\end{array}\right) T^{-1}
$$

where $\bar{A}$ is $(n-r) \times(n-r)$ matrix, whose eigenvalues have negative real parts. Then

$$
\exp (A t)=T\left(\begin{array}{cc}
\exp (\bar{A} t) & 0 \\
0 & E
\end{array}\right) T^{-1} \quad \text { or } \quad \exp (A t) T=T\left(\begin{array}{cc}
\exp (\bar{A} t) & 0 \\
0 & E
\end{array}\right) .
$$

Let the matrix $T$ also be presented in a block form $T=\left[T_{1} T_{2}\right]$, where $T_{1}$ is $n \times(n-r)$ matrix and $T_{2}$ is $n \times r$ matrix. Then $\exp (A t) T_{1}=T_{1} \exp (\bar{A} t)$ and $\exp (A t) T_{2}=T_{2}$.

A solution of system (2.3) under $k=0$ has the form

$$
\Pi_{0}^{i}\left(\nu_{i}\right)=X\left(\nu_{i}\right) c_{0}^{i}, \quad c_{0}^{i} \in \mathbf{R}^{n} .
$$

In order to realize a condition $\Pi_{0}\left(\nu_{i}\right) \rightarrow 0$ under $\varepsilon \rightarrow 0$ the last $r$ components of the vector $c_{0}^{i}$ are taken to be equal to zero. The solution of (2.3) under $k=0$ becomes the following

$$
\Pi_{0}^{i}\left(\nu_{i}\right)=X_{n-r}\left(\nu_{i}\right) c_{0}^{i}, \quad c_{0}^{i} \in \mathbf{R}^{n-r},
$$

where $X_{n-r}\left(\nu_{i}\right)=\exp \left(A \nu_{i}\right) T_{1}$ is $n \times(n-r)$ matrix. The solution of (2.3) under $k=1,2, \cdots$ is

$$
\Pi_{k}^{i}\left(\nu_{i}\right)=X_{n-r}\left(\nu_{i}\right) c_{k}^{i}+\int_{0}^{\infty} K\left(\nu_{i}, s\right) f_{k}^{i}(s) d s,
$$

where

$$
K\left(\nu_{i}, s\right)=\left\{\begin{array}{cc}
X\left(\nu_{i}\right) P X^{-1}(s), & 0 \leq s \leq \nu_{i}<\infty \\
-X\left(\nu_{i}\right)(I-P) X^{-1}(s), & 0 \leq \nu_{i} \leq s<\infty
\end{array}\right.
$$

$P$ is a spectral projector of the matrix $A$ on the left semi-plane.

A choice of $K\left(\nu_{i}, s\right)$ guarantees that the partial solutions of $(2.3)$ are bounded exponentially.

For definition of the vector-functions $\alpha_{k}^{i}(t)$ the form of $x_{k}^{i}(t)$ from (2.4) is substituted in the condition of solvability (I) and the following systems are obtained

$$
\bar{D} \dot{\alpha}_{k}^{i}(t)=B(t) \alpha_{k}^{i}(t)+g_{k}^{i}(t)=0, \quad k=0,1,2, \cdots, \quad i=\overline{1, p},
$$


where

$$
\begin{aligned}
& \bar{D}=P_{A^{*}}^{r} P_{A}^{r}, g_{k}^{i}(t)=\left\{\begin{array}{ll}
-P_{A^{*}}^{r}\left(L A^{+} \varphi_{i}\right)(t), & k=0 \\
-P_{A^{*}}^{r}\left(L A^{+}\left(L x_{k-1}^{i}\right)(t)\right)(t), & k=1,2, \ldots
\end{array},\right. \\
& B(t)=P_{A^{*}}^{r} A_{1}(t) P_{A}^{r} .
\end{aligned}
$$

According to condition $\mathrm{H} 1 \operatorname{rank} A=r$. It is easy to prove $\operatorname{rank} \bar{D}=r$.

A general solution of (2.6) in every subinterval $\left.] \tau_{i-1}, \tau_{i}\right], i=\overline{1, p+1}$ is

$$
\alpha_{k}^{i}(t)=\Phi(t) \Phi^{-1}\left(\tau_{i-1}\right) \eta_{k}^{i}+\int_{\tau_{i-1}}^{t} \Phi(t) \Phi^{-1}(s) \bar{D}^{-1} g_{k}^{i}(s) d s,
$$

where $\Phi(t)$ is $r \times r$ fundamental matrix of solutions of the homogeneous system $\dot{x}=\bar{D}^{-1} B(t) x$ and $\eta_{k}^{i}$ is $r$-dimensional unknown constant vector.

The initial and impulse conditions will be used for definition of unknown constant vectors $\eta_{k}^{i}$ and $c_{k}^{i}$. Because of the generalized character of the impulsive and initial conditions, the solution $x^{i}(\varepsilon, t)$ in every subinterval $\left(\tau_{i-1}, \tau_{i}\right], i=\overline{1, p+1}$ depends on an arbitrary constant vector $\xi_{i}$ under successive gradation from interval to interval. These constants take part in the elements of the singular series and also in the elements of the regular series. Therefore in the interval $\left(\tau_{i-1}, \tau_{i}\right]$ it is got accumulation of the constants $\xi_{1}, \xi_{2}, \cdots, \xi_{i}$ in the solution $x^{i}(\varepsilon, t)$. A dependence between constants $\xi_{i}$, $i=\overline{1, p+1}$ exists because of the recurrent relation between the elements $x_{k}^{i}(t)$ of the regular series and between the elements of the singular series. Difficulties are obvious if we work by successive gradation from interval to interval. For this reason a modification of the problem (1.1-1.3) similar to this one in [3] is needed.

The following denotations are introduced.

$$
\begin{aligned}
& Q_{0}=\left[D \Theta_{1} \cdots \Theta_{p}\right]^{T}, Q_{1}=\left[\Theta_{0} N_{1} \Theta_{2} \ldots \Theta_{p}\right]^{T}, \ldots, Q_{i}=\left[\Theta_{0} \cdots \Theta_{i-1} N_{i} \Theta_{i+1} \cdots \Theta_{p}\right]^{T}, \ldots \\
& Q_{p}=\left[\begin{array}{llll}
\Theta_{0} & \cdots & \Theta_{p-1} & N_{p}
\end{array}\right]^{T} \text {, }
\end{aligned}
$$



$$
\begin{aligned}
& R_{p}=\left[\begin{array}{llll}
\Theta_{0} & \cdots & \Theta_{p-1} & M_{p}
\end{array}\right]^{T}, h=\left[\begin{array}{llll}
v & h_{1} & \cdots & h_{p}
\end{array}\right]^{T} \text {, }
\end{aligned}
$$

where $Q_{i}, i=\overline{0, p}, R_{i}, i=\overline{1, p}$ are $\nu \times n$ matrices, $\nu=s+k_{1}+k_{2}+\cdots+k_{p}, \Theta_{0}$ is $s \times n$ matrix with zero elements, $\Theta_{i}, i=1,2, \cdots p$, are $k_{i} \times n$ matrices with zero elements, $h$ is $\nu$-dimensional vector. Then the initial and impulse conditions are rewritten as follows

$$
\sum_{i=0}^{p} Q_{i} x^{i+1}\left(\tau_{i}, \varepsilon\right)+\sum_{i=1}^{p} R_{i} x^{i}\left(\tau_{i}, \varepsilon\right)=h .
$$

The coefficients before identical powers of $\varepsilon$ are equalized in (2.8). Then

$$
\begin{aligned}
& \sum_{i=0}^{p} Q_{i}\left(x_{0}^{i+1}\left(\tau_{i}\right)+\Pi_{0}^{i+1}(0)\right)+\sum_{i=1}^{p} R_{i}\left(x_{0}^{i}\left(\tau_{i}\right)+\Pi_{0}^{i}\left(\frac{\tau_{i}-\tau_{i-1}}{\varepsilon}\right)\right)=h, \\
& \sum_{i=0}^{p} Q_{i}\left(x_{k}^{i+1}\left(\tau_{i}\right)+\Pi_{k}^{i+1}(0)\right)+\sum_{i=1}^{p} R_{i}\left(x_{k}^{i}\left(\tau_{i}\right)+\Pi_{k}^{i}\left(\frac{\tau_{i}-\tau_{i-1}}{\varepsilon}\right)\right)=0, \\
& k=1,2,3, \cdots
\end{aligned}
$$


In system (2.9) under $k=0$ are substituted $x_{0}^{i}(t)$ and $\Pi_{0}^{i}\left(\nu_{i}\right), i=\overline{1, p+1}$ from $(2.4)$ and (2.5), respectively. Then system (2.9) under $k=0$ takes the form

$$
\sum_{i=1}^{p+1}\left(l_{i} \alpha_{0}^{i}(\cdot)+D_{i}(\varepsilon) c_{0}^{i}\right)=h+\sum_{i=0}^{p} Q_{i} A^{+} \varphi_{i+1}\left(\tau_{i}\right)+\sum_{i=1}^{p} R_{i} A^{+} \varphi_{i}\left(\tau_{i}\right),
$$

where

$$
l_{i} x(\cdot)=Q_{i-1} P_{A}^{r} x\left(\tau_{i-1}\right)+R_{i} P_{A}^{r} x\left(\tau_{i}\right), i=\overline{1, p}, l_{p+1} x(\cdot)=Q_{p} P_{A}^{r} x\left(\tau_{i-1}\right)
$$

are $\nu \times r$ vector functionals,

$$
D_{i}(\varepsilon)=Q_{i-1} X_{n-r}(0)+R_{i} X_{n-r}\left(\frac{\tau_{i}-\tau_{i-1}}{\varepsilon}\right), i=\overline{1, p}, D_{p+1}(\varepsilon)=Q_{p} X_{n-r}(0)
$$

are $(\nu \times(n-r))$ matrices.

Let $\bar{D}(\varepsilon)=\left[D_{1}(\varepsilon) \cdots D_{p+1}(\varepsilon)\right]$ be a $(\nu \times((p+1)(n-r)))$ matrix, $l(\cdot)=\left[l_{1}(\cdot) \cdots l_{p+1}(\cdot)\right]$ is $(\nu \times((p+1) r))$ a vector functional, $\bar{h}_{0}=h+\sum_{i=0}^{p} Q_{i} A^{+} \varphi_{i+1}\left(\tau_{i}\right)+\sum_{i=1}^{p} R_{i} A^{+} \varphi_{i}\left(\tau_{i}\right)$ is a $\nu$-dimensional vector, $\alpha_{0}(t)=\left[\alpha_{0}^{1}(t) \cdots \alpha_{0}^{p+1}(t)\right]^{T}$ is a $(p+1) r$-dimensional vector, $c_{0}=\left[c_{0}^{1} \cdots c_{0}^{p+1}\right]^{T}$ is a $(p+1)(n-r)$-dimensional vector.

With these notations system (2.10) may be written as follows

$$
l \alpha_{0}(\cdot)=\bar{h}-\bar{D}(\varepsilon) c_{0} .
$$

In the last equality are substituted $\alpha_{0}^{i}(t), i=\overline{1, p+1}$ from (2.7). Then we obtain

$$
P \eta_{0}+\bar{D}(\varepsilon) c_{0}=\overline{\bar{h}}_{0}
$$

where $P=\left[P_{1} \cdots P_{p+1}\right]$ is $(\nu \times(n+1) r)$ matrix, $P_{i}=l_{i} \Phi(\cdot) \Phi^{-1}\left(\tau_{i-1}\right), i=\overline{1, p+1}$ are $(\nu \times r)$ matrices, $\left.\overline{\bar{h}}_{0}=\bar{h}_{0}-\sum_{i=1}^{p+1} l_{i} \int_{\tau_{i-1}}^{(} \cdot\right) \Phi(\cdot) \Phi^{-1}(s) \bar{D}^{-1} g_{0}^{i}(s) d s$ is $\nu$-dimensional vector and $\eta_{0}=\left[\eta_{0}^{1} \cdots \eta_{0}^{p+1}\right]^{T}$ is $(p+1) r$-dimensional vector.

The matrix $\bar{D}(\varepsilon)$ has a structure $\bar{D}(\varepsilon)=\bar{D}_{0}+O\left(\varepsilon^{q} \exp \left(-\frac{\alpha}{\varepsilon}\right)\right), q \in N, \alpha$ is a positive constant, $\bar{D}_{0}$ is $(\nu \times(p+1)(n-r))$ matrix with constant elements. The exponentially small elements in the matrix $\bar{D}(\varepsilon)$ are rejected and system (2.12) takes the form

$$
M\left[\begin{array}{l}
\eta_{0} \\
c_{0}
\end{array}\right]=\overline{\bar{h}}_{0}
$$

where $M=\left[P \bar{D}_{0}\right]$ is $(\nu \times(p+1) n)$ matrix. Let the following condition be fulfilled.

H6: $\operatorname{rank} M=m_{1} \leq \min (\nu,(p+1) n)$.

Then system (2.13) has a solution

$$
\left[\begin{array}{l}
\eta_{0} \\
c_{0}
\end{array}\right]=P_{M}^{k} \xi_{0}+M^{+} \overline{\bar{h}}_{0}
$$

if and only if 
H7: $P_{M^{*}}^{d} \overline{\bar{h}}_{0}=0$.

$P_{M}^{k}$ designates a matrix consisting of $k=(p+1)-m_{1}$ linear independent columns of the matrix projector $P_{M}, P_{M}: \mathbf{R}^{(p+1) n} \rightarrow \operatorname{ker} M$ and $P_{M^{*}}^{d}$ denotes a matrix consisting of $d=\nu-m_{1}$ linear independent rows of the matrix projector $P_{M^{*}}, P_{M^{*}}: \mathbf{R}^{\nu} \rightarrow$ $\operatorname{ker} M^{*}, \xi_{0} \in \mathbf{R}^{k}$ and $M^{+}$is a unique Moore-Penrose inverse matrix of the matrix $M$.

Let $(p+1) r=\bar{r}$ and $(p+1)(n-r)=\bar{n}$. Then

$$
\eta_{0}=\left[P_{M}^{k}\right]_{\bar{r}} \xi_{0}+\left[M^{+} \overline{\bar{h}}_{0}\right]_{\bar{r}}, \quad c_{0}=\left[P_{M}^{k}\right]_{\bar{n}} \xi_{0}+\left[M^{+} \overline{\bar{h}}_{0}\right]_{\bar{n}},
$$

where index $\bar{r}$ means the first $\bar{r}$ rows of the matrix $P_{M}^{k}$ and the vector $M^{+} \overline{\bar{h}}_{0}$ and index $\bar{n}$ means the last $\bar{n}$ rows of the matrix $P_{M}^{k}$ and the vector $M^{+} \overline{\bar{h}}_{0}$.

According to denotations of $\eta_{0}$ and $c_{0}$ above, we obtain

$$
\eta_{0}^{i}=\left[P_{M}^{k}\right]_{\bar{r}}^{r_{i}} \xi_{0}+\left[M^{+} \overline{\bar{h}}_{0}\right]_{\bar{r}}^{r_{i}}, \quad c_{0}=\left[P_{M}^{k}\right]_{\bar{n}}^{n_{i}} \xi_{0}+\left[M^{+} \overline{\bar{h}}_{0}\right]_{\bar{n}}^{n_{i}}, \quad i=\overline{1, p+1},
$$

where the index $r_{i}$ means that under $i=1$ we take the first $r$ rows of the matrix $\left[P_{M}^{k}\right]_{\bar{r}}$ and the vector $\left[M^{+} \overline{\bar{h}}_{0}\right]_{\bar{r}}$, under $i=2$ the second $r$ rows of the same matrix and the same vector and etc. The index $n_{i}$ means that under $i=1$ we take the first $(n-r)$ rows of the matrix $\left[P_{M}^{k}\right]_{\bar{n}}$ and the vector $\left[M^{+} \overline{\bar{h}}_{0}\right]_{\bar{n}}$, under $i=2$ - the second $(n-r)$ rows and etc.

According to (2.15) the forms of $x_{0}^{i}(t)$ and $\Pi_{0}^{i}\left(\nu_{i}\right)$ become the following

$$
\begin{aligned}
x_{0}^{i}(t) & =\Phi_{r}^{r_{i}}(t) \xi_{0}+\bar{x}_{0}^{i}(t), \Pi_{0}^{i}\left(\nu_{i}\right)=X_{n-r}^{n_{i}}\left(\nu_{i}\right) \xi_{0}+\bar{\Pi}_{0}^{i}\left(\nu_{i}\right) i=\overline{1, p+1} \\
\Phi_{r}^{r_{i}}(t) & =P_{A}^{r} \Phi(t) \Phi^{-1}\left(\tau_{i-1}\right)\left[P_{M}^{k}\right]_{\bar{r}}^{r_{i}}, X_{n-r}^{n_{i}}\left(\nu_{i}\right)=X_{n-r}\left(\nu_{i}\right)\left[P_{M}^{k}\right]_{\bar{r}}^{r_{i}}, \\
\bar{\Pi}_{0}^{i}\left(\nu_{i}\right) & =X_{n-r}\left(\nu_{i}\right)\left[M^{+} \overline{\bar{h}}_{0}\right]_{\bar{n}}^{n_{i}} \\
\bar{x}_{0}^{i}(t) & =P_{A}^{r} \Phi(t) \Phi^{-1}\left(\tau_{i-1}\right)\left[M^{+} \overline{\bar{h}}_{0}\right]_{\bar{r}}^{r_{i}}+P_{A}^{r} \int_{\tau_{i-1}}^{t} \Phi(t) \Phi^{-1}(s) \bar{D}^{-1} g_{0}^{i}(s)-A^{+} \varphi_{i}(t) .
\end{aligned}
$$

On analogy of system (2.11) the following system is obtained

$$
l \alpha_{1}(\cdot)+\bar{D}(\varepsilon) c_{1}=\bar{h}_{1}(\varepsilon),
$$

where $\alpha_{1}(t)=\left[\alpha_{1}^{1}(t) \cdots \alpha_{1}^{p+1}(t)\right]^{T}$ is $(p+1) r$-dimensional vector, $c_{1}=\left[c_{1}^{1} \cdots c_{1}^{p+1}\right]^{T}$ is $(p+1)(n-r)$-dimensional vector.

Keeping in mind the expressions from $(2.16)$, the $\nu$-dimensional vector $\bar{h}_{1}(\varepsilon)$ may be written as follows

$$
\bar{h}_{1}(\varepsilon)=\left(A_{11}+A_{12}(\varepsilon)\right) \xi_{0}+a_{1}(\varepsilon)
$$

where

$$
A_{11}=-\sum_{i=0}^{p+1} Q_{i} A^{+}\left(L \Phi_{r}^{r_{i+1}}\right)\left(\tau_{i}\right)-\sum_{i=1}^{p} R_{i} A^{+}\left(L \Phi_{r}^{r_{i}}\right)\left(\tau_{i}\right),
$$




$$
\begin{aligned}
& A_{12}(\varepsilon)=-\sum_{i=0}^{p+1} Q_{i} \int_{0}^{\infty} K(0, s) A_{1}\left(\tau_{i}\right) X_{n-r}^{n_{i+1}}(s) d s- \\
& \quad-\sum_{i=1}^{p} R_{i} \int_{0}^{\infty} K\left(\frac{\tau_{i}-\tau_{i-1}}{\varepsilon}, s\right) A_{1}\left(\tau_{i}\right) X_{n-r}^{n_{i}}(s) d s \\
& a_{1}(\varepsilon)=-\sum_{i=0}^{p+1} Q_{i} A^{+}\left(L \bar{x}_{0}^{i+1}\right)\left(\tau_{i}\right)-\sum_{i=1}^{p} R_{i} A^{+}\left(L \bar{x}_{0}^{i}\right)\left(\tau_{i}\right)- \\
& -\sum_{i=0}^{p+1} Q_{i} \int_{0}^{\infty} K(0, s) A_{1}\left(\tau_{i}\right) \bar{\Pi}_{0}^{i+1}(s) d s-\sum_{i=1}^{p} R_{i} \int_{0}^{\infty} K\left(\frac{\tau_{i}-\tau_{i-1}}{\varepsilon}, s\right) A_{1}\left(\tau_{i}\right) \bar{\Pi}_{0}^{i}(s) d s .
\end{aligned}
$$

According to (2.6) the functions $\alpha_{1}^{i}(t), i=\overline{1, p+1}$ are defined from the systems

$$
\bar{D} \dot{\alpha}_{1}^{i}(t)=B(t) \alpha_{1}^{i}(t)+g_{1}^{i}(t), i=\overline{1, p+1} .
$$

The functions $g_{1}^{i}(t), i=\overline{1, p+1}$ are presented in the form

$$
g_{1}^{i}(t)=B_{11}^{i}(t) \xi_{0}+b_{1}^{i}(t),
$$

where $B_{11}^{i}(t)=-P_{A^{*}}^{r}\left(L A^{+}\left(L \Phi_{r}^{r_{i}}\right)(t)\right)(t), b_{1}^{i}(t)=-P_{A^{*}}^{r}\left(L A^{+}\left(L \bar{x}_{0}^{i}\right)(t)\right)(t)$.

Analogously to system (2.13) the following system is obtained

$$
M\left[\begin{array}{l}
\eta_{1} \\
c_{1}
\end{array}\right]=\overline{\bar{h}}_{1}(\varepsilon)
$$

where $\eta_{1}=\left(\eta_{1}^{1} \cdots \eta_{1}^{p+1}\right)^{T}$ is $(p+1) r$-dimensional vector and

$$
\overline{\bar{h}}_{1}(\varepsilon)=\bar{A}_{11}(\varepsilon) \xi_{0}+\bar{a}_{1}(\varepsilon),
$$

$\bar{A}_{11}(\varepsilon)=A_{11}+A_{12}(\varepsilon)-\sum_{i=1}^{p+1} l_{i} \int_{\tau_{i-1}}^{(\cdot)} \Phi(\cdot) \Phi^{-1}(s) B_{11}^{i}(s) d s$,

$\bar{a}_{1}(\varepsilon)=a_{1}(\varepsilon)-\sum_{i=1}^{p+1} l_{i} \int_{\tau_{i-1}}^{(\cdot)} \Phi(\cdot) \Phi^{-1}(s) b_{1}^{i}(s) d s$.

System (2.20) has solution

$$
\eta_{1}^{i}=\left[P_{M}^{k}\right]_{\bar{r}}^{r_{i}} \xi_{1}+\left[M^{+} \overline{\bar{h}}_{1}\right]_{\bar{r}}^{r_{i}}, c_{1}^{i}=\left[P_{M}^{k}\right]_{\bar{n}}^{n_{i}} \xi_{1}+\left[M^{+} \overline{\bar{h}}_{1}\right]_{\bar{n}}^{n_{i}}, i=\overline{1, p+1},
$$

if and only if $P_{M^{*}}^{d} \overline{\bar{h}}_{1}=0$.

Keeping in mind the form of $\overline{\bar{h}}_{1}(\varepsilon)$ from $(2.21)$, the last condition may be written as follows

$$
R(\varepsilon) \xi_{0}=\overline{\bar{a}}_{1}(\varepsilon),
$$

where $R(\varepsilon)=P_{M^{*}}^{d} \bar{A}_{11}(\varepsilon)$ is $d \times \nu$ matrix and $\overline{\bar{a}}_{1}(\varepsilon)=-P_{M^{*}}^{d} \bar{a}_{1}(\varepsilon)$ is d-dimensional vector.

The matrix $R(\varepsilon)$ has a structure $R(\varepsilon)=R_{0}+O\left(\varepsilon^{s} \exp \left(-\frac{\alpha}{\varepsilon}\right)\right)$ and the vector $\overline{\bar{a}}_{1}(\varepsilon)$ has a structure $\overline{\bar{a}}_{1}(\varepsilon)=\overline{\bar{a}}_{10}+O\left(\varepsilon^{q} \exp \left(-\frac{\alpha}{\varepsilon}\right)\right), s, q \in N, \alpha$-positive constant, $R_{0}-d \times \nu$ constant matrix, $\overline{\bar{a}}_{10^{-}} d$-dimensional constant vector. 
The exponentially small elements in $R(\varepsilon)$ and $\overline{\bar{a}}_{1}(\varepsilon)$ are rejected and system $(2.23)$ takes the form

$$
R_{0} \xi_{0}=\overline{\bar{a}}_{10}
$$

Let the following condition be fulfilled

H8: $\operatorname{rank} R_{0}=\nu<d$.

Then system (2.24) has a unique solution

$$
\xi_{0}=R_{0}^{+} \overline{\bar{a}}_{10},
$$

if and only if $P_{R_{0}^{*}} P_{M^{*}}^{d} \overline{\bar{a}}_{10}=0$.

The last requirement is always fulfilled if the following condition is real

H9: $P_{R_{0}^{*}} P_{M^{*}}^{d}=0$.

According to (2.25), the equalities (2.16) take the representation

$x_{0}^{i}(t)=\Phi_{r}^{r_{i}}(t) R_{0}^{+} \overline{\bar{a}}_{10}+\bar{x}_{0}^{i}(t), \Pi_{0}^{i}\left(\nu_{i}\right)=X_{n-r}^{n_{i}}\left(\nu_{i}\right) R_{0}^{+} \overline{\bar{a}}_{10}+\bar{\Pi}_{0}^{i}\left(\nu_{i}\right), i=\overline{1, p+1}$.

From (2.4), (2.5) and (2.22) the following is obtained

$$
x_{1}^{i}(t)=\Phi_{r}^{r_{i}}(t) \xi_{1}+\bar{x}_{1}^{i}(t), \Pi_{1}^{i}\left(\nu_{i}\right)=X_{n-r}^{n_{i}}\left(\nu_{i}\right) \xi_{1}+\bar{\Pi}_{1}^{i}\left(\nu_{i}\right), i=\overline{1, p+1},
$$

where $\bar{x}_{1}^{i}(t)=P_{A}^{r} \Phi(t) \Phi^{-1}\left(\tau_{i-1}\right)\left[M^{+} \bar{h}_{1}\right]_{\bar{r}}^{r_{i}}+P_{A}^{r} \int_{\tau_{i-1}}^{t} \Phi(t) \Phi^{-1}(s) \bar{D}^{-1} g_{1}^{i}(s) d s+A^{+}\left(L x_{0}^{i}\right)(t)$, $\bar{\Pi}_{1}^{i}\left(\nu_{i}\right)=X_{n-r}\left(\nu_{i}\right)\left[M^{+} \overline{\bar{h}}_{1}\right]_{\bar{n}}^{n_{i}}+\int_{0}^{\infty} K(\tau, s) A_{1}\left(\tau_{i}\right) \Pi_{0}^{i}(s) d s$.

Analogously to system (2.11) the following system is obtained

$$
l \alpha_{2}(\cdot)+\bar{D}(\varepsilon) c_{2}=\bar{h}_{2}(\varepsilon),
$$

where $\alpha_{2}(t)=\left(\alpha_{2}^{1}(t) \cdots \alpha_{2}^{p+1}(t)\right)^{T}$ is $(p+1) r$-dimensional vector, $c_{2}=\left(c_{2}^{1} \cdots c_{2}^{p+1}\right)^{T}$ is

$(p+1)(n-r)$ - dimensional vector and $\bar{h}_{2}(\varepsilon)=\left(A_{11}+A_{12}(\varepsilon)\right) \xi_{1}+a_{2}(\varepsilon)$ is $\nu$-dimensional vector, $a_{2}(\varepsilon)=-\sum_{i=0}^{p} Q_{i} A^{+}\left(L \bar{x}_{1}^{i+1}\right)\left(\tau_{i}\right)-\sum_{i=1}^{p} R_{i} A^{+}\left(L \bar{x}_{1}^{i}\right)\left(\tau_{i}\right)-$

$-\sum_{i=0}^{p} Q_{i} \int_{0}^{\infty} K(0, s)\left(A_{1}\left(\tau_{i}\right) \bar{\Pi}_{1}^{i+1}(s)+A_{1}^{\prime}\left(\tau_{i}\right) s \Pi_{0}^{i+1}(s)\right) d s-$

$-\sum_{i=1}^{p} R_{i} \int_{0}^{\infty} K\left(\frac{\tau_{i}-\tau_{i-1}}{\varepsilon}, s\right)\left(A_{1}\left(\tau_{i}\right) \bar{\Pi}_{1}^{i}(s)+A_{1}^{\prime}\left(\tau_{i}\right) s \Pi_{0}^{i}(s)\right) d s$.

According to (2.7), the functions $\alpha_{2}^{i}(t), i=\overline{1, p+1}$ have the form

$$
\alpha_{2}^{i}(t)=\Phi(t) \Phi^{-1}\left(\tau_{i-1}\right) \eta_{2}^{i}+\int_{\tau_{i-1}}^{t} \Phi(t) \Phi^{-1}(s) \bar{D}^{-1} g_{2}^{i}(s) d s,
$$

where $g_{2}^{i}(t)$ has the form

$$
\begin{aligned}
& g_{2}^{i}(t)=B_{11}^{i}(t) \xi_{1}+b_{2}^{i}(t), \\
& b_{2}^{i}(t)=-P_{A^{*}}^{r}\left(L A^{+}\left(L \bar{x}_{1}^{i}\right)(t)\right)(t) .
\end{aligned}
$$

A system

$$
M\left[\begin{array}{l}
\eta_{2} \\
c_{2}
\end{array}\right]=\bar{h}_{2}\left(\varepsilon, \xi_{1}\right)
$$


is obtained analogously to system (2.11). From solvability condition $P_{M^{*}}^{d} \bar{h}_{2}\left(\varepsilon, \xi_{1}\right)=0$ of system (2.30) and by analogy to (2.23), the following system is obtained

$$
R(\varepsilon) \xi_{1}=\overline{\bar{a}}_{2}(\varepsilon),
$$

where $\overline{\bar{a}}_{2}(\varepsilon)=-P_{M^{*}}^{d} \bar{a}_{2}(\varepsilon), \bar{a}_{2}(\varepsilon)=a_{2}(\varepsilon)-\sum_{i=1}^{p+1} l_{i} \int_{\tau_{i-1}}^{(\cdot)} \Phi(\cdot) \Phi^{-1}(s) \bar{D}^{-1} b_{2}^{i}(s) d s$, and $\overline{\bar{a}}_{2}(\varepsilon)=\overline{\bar{a}}_{20}+O\left(\varepsilon^{s} \exp \left(-\frac{\alpha}{\varepsilon}\right)\right) s \in N, \alpha$ - positive constant, $\overline{\bar{a}}_{20}$ - $d$-dimensional vector.

The exponentially small elements in $R(\varepsilon)$ and $\overline{\bar{a}}_{2}(\varepsilon)$ are rejected and system $(2.31)$ takes the form

$$
R_{0} \xi_{1}=\overline{\bar{a}}_{20}
$$

The last system under condition $\mathrm{H} 8$ has a unique solution

$$
\xi_{1}=R_{0}^{+} \overline{\bar{a}}_{20} .
$$

if and only if condition H9 is fulfilled. The last equality is substituted in (2.27). Then the following expressions for $x_{1}^{i}(t)$ and $\Pi_{1}^{i}\left(\nu_{i}\right)$ are obtained

$$
x_{1}^{i}(t)=\Phi_{r}^{r_{i}}(t) R_{0}^{+} \overline{\bar{a}}_{20}+\bar{x}_{1}^{i}(t), \Pi_{1}^{i}\left(\nu_{i}\right)=X_{n-r}^{n_{i}} R_{0}^{+} \overline{\bar{a}}_{20}+\bar{\Pi}_{1}^{i}\left(\nu_{i}\right), i=\overline{1, p+1} .
$$

Analogously to the statement above for $k=2,3, \cdots$ the following is obtained

$$
\begin{gathered}
x_{k}^{i}(t)=\Phi_{r}^{r_{i}}(t) R_{0}^{+} \overline{\bar{a}}_{k+1,0}+\bar{x}_{k}^{i}(t), \Pi_{k}^{i}\left(\nu_{i}\right)=X_{n-r}^{n_{i}} R_{0}^{+} \overline{\bar{a}}_{k+1,0}+\bar{\Pi}_{k}^{i}\left(\nu_{i}\right), i=\overline{1, p+1 .} \\
\bar{x}_{k}^{i}(t)=P_{A}^{r} \Phi(t) \Phi^{-1}\left(\tau_{i-1}\right)\left[M^{+} \overline{\bar{h}}_{k}\right]_{\bar{r}}^{r_{i}}+P_{A}^{r} \int_{\tau_{i-1}}^{t} \Phi(t) \Phi^{-1}(s) \bar{D}^{-1} g_{k}^{i}(s) d s+A^{+}\left(L x_{k-1}^{i}\right)(t), \\
\bar{\Pi}_{k}^{i}\left(\nu_{i}\right)=X_{n-r}\left(\nu_{i}\right)\left[M^{+} \overline{\bar{h}}_{k}\right]_{\bar{n}}^{n_{i}}+\int_{0}^{\infty} K(\tau, s) \sum_{j=0}^{k-1} \frac{A_{1}^{k-j-1}\left(\tau_{i}\right)}{(k-j-1) !} s^{k-j-1} \Pi_{j}^{i}(s) d s, \\
\overline{\bar{a}}_{k}(\varepsilon)=-P_{M^{*}}^{d} \bar{a}_{k}(\varepsilon), \bar{a}_{k}(\varepsilon)=a_{k}(\varepsilon)-\sum_{i=1}^{p+1} l_{i} \int_{\tau_{i-1}}^{(\cdot)} \Phi(\cdot) \Phi^{-1}(s) \bar{D}^{-1} b_{k}^{i}(s) d s, \\
b_{k}^{i}(t)=-P_{A^{*}}^{r}\left(L A^{+}\left(L \bar{x}_{k-1}^{i}\right)(t)\right)(t), a_{k}(\varepsilon)=-\sum_{i=0}^{p} Q_{i} A^{+}\left(L \bar{x}_{k-1}^{i+1}\right)\left(\tau_{i}\right)- \\
-\sum_{i=1}^{p} R_{i} A^{+}\left(L \bar{x}_{k-1}^{i}\right)\left(\tau_{i}\right)-\sum_{i=0}^{p} Q_{i} \int_{0}^{\infty} K(0, s)\left(\sum_{j=0}^{k-2} \frac{A_{1}^{(k-j-1)}\left(\tau_{i}\right)}{(k-j-1) !} s^{k-j-1} \Pi_{j}^{i+1}(s)+\right. \\
\left.+A_{1}\left(\tau_{i}\right) \bar{\Pi}_{k-1}^{i+1}(s)\right) d s-\sum_{i=1}^{p} R_{i} \int_{0}^{\infty} K\left(\frac{\tau_{i}-\tau_{i-1}}{\varepsilon}, s\right)\left(\sum_{j=0}^{k-2} \frac{A_{1}^{(k-j-1)}\left(\tau_{i}\right)}{(k-j-1) !} s^{k-j-1} \Pi_{j}^{i+1}(s)+\right. \\
\left.+A_{1}\left(\tau_{i}\right) \bar{\Pi}_{k-1}^{i}(s)\right) d s, \overline{\bar{h}}_{k}(\varepsilon)=\bar{A}_{11}(\varepsilon) \xi_{k-1}+\bar{a}_{k}(\varepsilon), \bar{h}_{k}(\varepsilon)=\left(A_{11}+A_{12}(\varepsilon)\right) \xi_{k-1}+a_{k}(\varepsilon), \\
g_{k}^{i}(t)=B_{11}^{i}(t) R_{0}^{+} \overline{\bar{a}}_{k+1,0}+b_{k}^{i}(t) .
\end{gathered}
$$

Let

$$
u(t, \varepsilon)=x(t, \varepsilon)-X_{n}(t, \varepsilon),
$$

where $x(t, \varepsilon)$ is the exact solution of (1.1), (1.2), (1.3) and

$$
X_{n}(t, \varepsilon)=\sum_{k=0}^{n} \varepsilon^{k}\left(x_{k}^{i}(t)+\Pi_{k}^{i} x\left(\nu_{i}\right)\right), \quad i=\overline{1, p+1} .
$$


It is easy to show that under some conditions $\|u(t, \varepsilon)\| \leq c \varepsilon^{n+1}$, using the scheme of proof in [8], [1] with the necessary changes originating from the generalized initial and impulse conditions.

On this way the following theorem is proved.

Theorem 1: Let the conditions $\mathrm{H1}-\mathrm{H}_{4}, \mathrm{H} 6$ and $\mathrm{H} 8$ be fulfilled. The initial impulsive problem (1.1),(1.2), (1.3) has a unique asymptotic expansion of the solution in the form (2.1). The coefficients of the regular and singular series have the representation (2.26), under $k=0$ and (2.32), (2.33) under $k=1,2,3, \cdots$ if and only if $\varphi(t)$ satisfies the condition $\mathrm{H} 5$ and $v, h_{i}, i=\overline{1, p}$ satisfy $\mathrm{H}^{\mathrm{r}}$ and $\mathrm{H}$.

The next bound is true for the boundary functions

$$
\left\|\Pi_{k}^{i}\left(\nu_{i}\right)\right\| \leq \sigma \exp \left(-\kappa \nu_{i}\right), \quad i=\overline{1, p+1}, \quad k=0,1, \cdots,
$$

where $\sigma$ and $\kappa$ are positive constants.

Remark 1: Let, instead of H6, the following condition be fulfilled

H10: $\nu=(p+1) n, \operatorname{rank} M=m_{1}<\min (\nu,(p+1) n)$.

Then $\operatorname{rank} P_{M}=\operatorname{rank} P_{M^{*}}=\nu-m_{1}=k=d$. The matrix $\operatorname{rank} P_{M^{*}}$ is $k \times \nu$ matrix and the matrix $R_{0}$ is a rectangular matrix. According to $H 8(d=k)$, system (2.23) is always solvable as condition $H 9$ is always real and $R_{0}^{+}=R_{0}^{-1}$. The solution of problem (1.1),(1.2), (1.3) is presented in series (2.1) whose coefficients have the form (2.26), (2.32), (2.33)

Remark 2: Let instead of H6 the following condition be fulfilled

H11: $\nu=(p+1) n, \operatorname{det} M \neq 0$.

Then systems (2.13), (2.30) are always solvable, $P_{M^{*}}=0, M^{+}=M^{-1}$. The coefficients of the formally asymptotic solution of the problem (1.1),(1.2), (1.3) have the representation

$$
x_{k}^{i}(t)=\bar{x}_{k}^{i}(t), \Pi_{k}^{i}\left(\nu_{i}\right)=\bar{\Pi}_{k}^{i}\left(\nu_{i}\right), k=0,1,2, \cdots, i=\overline{1, p+1} .
$$

\section{Example}

Let $t \in[0,2], t \neq \tau_{1}, \tau_{1}=1$ and problem (1.1-1.3) have the following coefficients:

$$
\begin{aligned}
& A=\left(\begin{array}{rr}
-1 & 2 \\
1 & -2
\end{array}\right), \quad A_{1}(t)=\left(\begin{array}{rr}
-t & 2 t+1 \\
2 t & -4 t+2
\end{array}\right), \quad \varphi(t)=\left\{\begin{array}{cc}
\left(\begin{array}{cc}
1 & -1 \\
0, & t \in[0,1
\end{array}\right), & t \in(1,2]
\end{array}\right. \text { ， } \\
& D=\left(\begin{array}{ll}
2 & 6
\end{array}\right), \quad v=1, \quad N_{1}=\left(\begin{array}{ll}
0 & 0 \\
1 & 3 \\
2 & 1
\end{array}\right), \quad M_{1}=\left(\begin{array}{cc}
-1 & 2 \\
0 & 0 \\
1 & -2
\end{array}\right), \quad h_{1}=\left(\begin{array}{c}
-1 \\
1 \\
4
\end{array}\right)
\end{aligned}
$$

Then

$$
A^{+}=\frac{1}{10}\left(\begin{array}{rr}
-1 & 1 \\
2 & -2
\end{array}\right), P_{A}^{1}=\frac{1}{5}\left(\begin{array}{l}
2 \\
1
\end{array}\right), P_{A^{*}}^{1}=\frac{1}{2}\left(\begin{array}{ll}
1 & 1
\end{array}\right)
$$


Obviously the requirement $\mathrm{H} 5$ is fulfilled. Further $\bar{D}=\frac{3}{10}, \bar{D}^{-1}=\frac{10}{3}, B(t)=\frac{3}{10}$, $\Phi(t)=e^{t}, \Phi^{-1}(t)=e^{-t}$,

$$
\begin{aligned}
& X(t)=\frac{1}{3}\left(\begin{array}{cc}
2+e^{-3 t} & 2-2 e^{-3 t} \\
1-e^{-3 t} & 1+2 e^{-3 t}
\end{array}\right), X^{-1}(t)=\frac{1}{3}\left(\begin{array}{cc}
2+e^{3 t} & 2-2 e^{3 t} \\
1-e^{3 t} & 1+2 e^{3 t}
\end{array}\right), \\
& X_{1}(t)=\left(\begin{array}{r}
1 \\
-1
\end{array}\right) e^{-3 t}, P=\frac{1}{3}\left(\begin{array}{rr}
1 & -2 \\
-1 & 2
\end{array}\right), I-P=\frac{1}{3}\left(\begin{array}{cc}
2 & 2 \\
1 & 1
\end{array}\right) \text {, } \\
& K\left(\nu_{i}, s\right)=\left\{\begin{array}{lr}
\frac{1}{3}\left(\begin{array}{rr}
e^{-3\left(\nu_{i}-s\right)} & -2 e^{-3\left(\nu_{i}-s\right)} \\
-e^{-3\left(\nu_{i}-s\right)} & 2 e^{-3\left(\nu_{i}-s\right)},
\end{array}\right) & 0 \leq s \leq \nu_{i}<\infty, \\
\frac{1}{3}\left(\begin{array}{cc}
2 & 2 \\
1 & 1
\end{array}\right), & 0 \leq \nu_{i} \leq s<\infty .
\end{array}\right. \\
& Q_{0}=\left(\begin{array}{ll}
2 & 6 \\
0 & 0 \\
0 & 0 \\
0 & 0
\end{array}\right), Q_{1}=\left(\begin{array}{ll}
0 & 0 \\
0 & 0 \\
1 & 3 \\
2 & 1
\end{array}\right), \quad R_{1}=\left(\begin{array}{cc}
0 & 0 \\
-1 & 2 \\
0 & 0 \\
1 & -2
\end{array}\right), \quad \bar{h}_{0}=\left(\begin{array}{c}
1 \\
-1 \\
1 \\
4
\end{array}\right) \text {, } \\
& P=\left(\begin{array}{ll}
2 & 0 \\
0 & 0 \\
0 & 1 \\
0 & 1
\end{array}\right), \bar{D}(\varepsilon)=\left(\begin{array}{cc}
-4 & 0 \\
-3 e^{-\frac{3}{\varepsilon}} & 0 \\
0 & -2 \\
3 e^{-\frac{3}{\varepsilon}} & 1
\end{array}\right), M=\left(\begin{array}{cccc}
2 & 0 & -4 & 0 \\
0 & 0 & 0 & 0 \\
0 & 1 & 0 & -2 \\
0 & 1 & 0 & 1
\end{array}\right) \text {. }
\end{aligned}
$$

$\overline{\bar{h}}_{0}=\left(\begin{array}{llll}3 & 0 & 1 & 3\end{array}\right)^{T}, P_{M^{*}}^{1} \overline{\bar{h}}_{0}=0$, i.e., the condition $\mathrm{H} 7$ is fulfilled.

Let $t \in[0,1]$, then $\nu_{1}=\frac{t}{\varepsilon}$ and in accordance with (2.7) for $\alpha_{0}^{1}(t)$ and $\alpha_{0}^{2}(t)$ the following is obtained

$$
\begin{gathered}
\alpha_{0}^{1}(t)=e^{t} \eta_{0}^{1}+\frac{1}{3} e^{t}+\frac{5}{3} t-\frac{1}{3}, \alpha_{0}^{2}(t)=e^{t-1} \eta_{0}^{2} \\
x_{0}^{1}(t)=\frac{1}{5}\left(\begin{array}{c}
2 \\
1
\end{array}\right) e^{t} \eta_{0}^{1}+\frac{1}{5}\left(\begin{array}{c}
2 \\
1
\end{array}\right)\left(\frac{1}{3} e^{t}+\frac{5}{3} t-\frac{1}{3}\right)-\frac{1}{5}\left(\begin{array}{r}
-1 \\
2
\end{array}\right), \Pi_{0}^{1}\left(\nu_{1}\right)=\left(\begin{array}{c}
1 \\
-1
\end{array}\right) e^{-3 \nu_{1}} c_{0}^{1},
\end{gathered}
$$

and under $t \in(1,2]$, then $\nu_{2}=\frac{t-1}{\varepsilon}$

$$
x_{0}^{2}(t)=\frac{1}{5}\left(\begin{array}{l}
2 \\
1
\end{array}\right) e^{t-1} \eta_{0}^{2}, \Pi_{0}^{2}\left(\nu_{2}\right)=\left(\begin{array}{c}
1 \\
-1
\end{array}\right) e^{-3 \nu_{2}} c_{0}^{2} .
$$

From (2.14) the following is obtained

Further

$$
\eta_{0}^{1}=\frac{2}{5} \xi_{0}+\frac{3}{10}, \eta_{0}^{2}=\frac{7}{3}, c_{0}^{1}=\frac{1}{5} \xi_{0}-\frac{3}{5}, c_{0}^{2}=\frac{2}{3} .
$$

$$
R(\varepsilon)=-\frac{2}{25} e+\frac{3}{\varepsilon} e^{-\frac{3}{\varepsilon}}, \overline{\bar{a}}_{1}(\varepsilon)=\frac{19}{150} e+\frac{24}{15}-\frac{9}{5} \frac{e^{\frac{-3}{\varepsilon}}}{\varepsilon} .
$$


Then $R_{0}=-\frac{2}{25} e$ and $\overline{\bar{a}}_{10}=\frac{19}{150} e+\frac{24}{15}$. In this case $R_{0}^{+}=R_{0}^{-1}=-\frac{25}{2 e}, \xi_{0}=-\frac{19}{12}-\frac{20}{e}$ and $P_{R_{0}^{*}}=0$, i.e., the condition H9 is fulfilled.

$$
x^{1}(t, \varepsilon)=-\frac{8}{5} e\left(\begin{array}{c}
2 \\
1
\end{array}\right) e^{t}+\left(\begin{array}{c}
10 t+1 \\
5 t-7
\end{array}\right)+\left(\begin{array}{c}
1 \\
-1
\end{array}\right)\left(-\frac{11}{12}-\frac{4}{e}\right) e^{-3 \frac{t}{\varepsilon}}+O(\varepsilon),
$$

$t \in[0,1]$.

$$
x^{2}(t, \varepsilon)=\frac{7}{15}\left(\begin{array}{l}
2 \\
1
\end{array}\right) e^{t-1}+\frac{2}{3}\left(\begin{array}{c}
1 \\
-1
\end{array}\right) e^{-3 \frac{t-1}{\varepsilon}}+O(\varepsilon),
$$

$t \in(1,2]$.

\section{REFERENCES}

[1] Bainov, D.D. and Covachev, V.: Impulsive Differential Equations with Small Parameter, World Scientific Publishing Co. Ptc. Ltd., Singapore, 1994.

[2] Halanaj, A. and Veskler, O.: Theory of Impulsive Systems, Moscow, Mir, 1971. (in Russian)

[3] Karandulov, L.I.: Generalized Cauchy problem for linear pulse differential systems, Mathematics and Education in Mathematics, Proceedings of Twenty-Eighth Spring Conference of the Union of Bulgarian Mathematicians, Montana, April 5 - 8, (1999), 120 127.

[4] NACheD, M.Z.: Generalized Inverse and Applications, Ed. by Nached M.Z., New Jork, San Francisco, London - Acad. Press, 1976.

[5] Penrose, R.: On best approximate solution on linear matrix equations, Proc. Cambridge Philos. Soc., 52, (1956), 17-19.

[6] Penrose, R.: A generalized inverse for matrices, Proc. Cambridge Philos. Soc., 51, (1955), 406-413.

[7] Samoilenko, A.M. and Perestyik, N.A.: Differential Equations with Impulse Actions, Kiev, 1987. (in Russian).

[8] Vasil'eva, A. B. and Butuzov, V. F.: Asymptotic Expansions of Solution of Singularly Perturbed Equations, Nauka, Moscow 1973. (in Russian)

[9] Vasil'eva, A. B. and Butuzov, V. F.: Singularly Perturbed Equations in Critical Case, Moscow State University, 1978. (in Russian). 